\title{
Treatment Response to Idiopathic Retroperitoneal Fibrosis-associated Hydronephrosis With a Focus on IgG4/IgG3 Serum Concentration Ratio
}

\author{
Su Jin Choi, M.D. ${ }^{1}$, Ji Seon Oh, M.D., Ph.D. ${ }^{2}$, Seokchan Hong, M.D., Ph.D. ${ }^{1}$, Chang-Keun Lee, M.D., Ph.D. ${ }^{1}$, \\ Bin Yoo, M.D., Ph.D. ${ }^{1}$, Bumsik Hong, M.D., Ph.D. ${ }^{3}$, Yong-Gil Kim, M.D., Ph.D. ${ }^{1}$ \\ ${ }^{1}$ Division of Rheumatology, Department of Internal Medicine, Asan Medical Center, University of Ulsan College of Medicine, ${ }^{2}$ Department of \\ Biomedical Informatics, Asan Medical Center, ${ }^{3}$ Department of Urology, Asan Medical Center, University of Ulsan College of Medicine, Seoul, \\ Korea
}

Objective. Hydronephrosis, a common complication of idiopathic retroperitoneal fibrosis (iRPF), may lead to poor renal outcomes unless resolved in a timely manner. IgG4-related diseases characterized by elevated serum IgG4 levels are responsible for a few iRPF cases. However, the underlying immunologic features of most iRPF cases have not been clearly defined, and these cases exhibit varied responses to medical treatment. Thus, we investigated the predictive factors for hydronephrosis-associated outcomes among iRPF patients. Methods. We retrospectively included 18 iRPF patients with hydronephrosis in a tertiary referral hospital from 2012 to 2019. Hydronephrosis improvement was assessed on images taken 6 months after diagnosis. Categorical variables were compared using chi-square or Fisher's exact test. Continuous variables were compared using Mann-Whitney U-test. Results. On follow-up images, 8 patients $(44.4 \%)$ showed an improvement in hydronephrosis. Patients with improvement more frequently had reverse serum IgG4/IgG3 ratio $(87.5 \%$ vs. $30 \%, p=0.025)$, abdominal aorta involvement $(87.5 \%$ vs. 30\%, $\mathrm{p}=0.025)$ and glucocorticoid treatment administration $(87.5 \%$ vs. 30\%, $\mathrm{p}=0.025$ ) than those without improvement. The proportion of elevated serum IgG4 level did not differ between the two groups. Even in the 14 cases with normal serum IgG4 levels, reverse serum IgG4/IgG3 ratio was more frequently observed in patients with improvement than in those without improvement ( $83.3 \%$ vs. $12.5 \%, p=0.026)$. Conclusion. The reverse serum IgG4/IgG3 ratio was associated with hydronephrosis improvement in iRPF patients, suggesting it to be a suitable serologic marker for predicting favourable responses to glucocorticoid treatment. (J Rheum Dis 2021;28:38-44)

Key Words. Immunoglobulins, Retroperitoneal fibrosis, Hydronephrosis, Glucocorticoids

\section{INTRODUCTION}

Retroperitoneal fibrosis (RPF) is a rare fibro-inflammatory disease in which inflammation occurs and fibrous tissues develop around the abdominal aorta and other retroperitoneal structures [1]. Ureteral involvement has been reported in $80 \% \sim 100 \%$ of the cases at presentation, occasionally causing hydronephrosis [2]. To prevent the progression to a chronic renal complication, early resolution of hydronephrosis is essential.
RPF is categorized as secondary or idiopathic causes [3]. Secondary causes of RPF include infection, drug use, malignancy, radiation therapy and abdominal operation. Idiopathic RPF (iRPF) accounts for two-thirds of the reported RPF cases and can be divided into two groups: IgG4-related RPF and non-IgG4-related RPF [4]. Pathological confirmation can help identify IgG4-related RPF and determine the treatment strategy. However, in many cases, it is difficult to obtain sufficient tissues owing to the location of fibrosis and high risks associated with open

\footnotetext{
Received : September 1, 2020, Revised : October 15, 2020, Accepted : October 28, 2020

Corresponding to : Yong-Gil Kim (1D http://orcid.org/0000-0002-8029-7355

Division of Rheumatology, Department of Internal Medicine, Asan Medical Center, University of Ulsan College of Medicine, 88 Olympic-ro 43-gil, Songpa-gu, Seoul 05505, Korea. E-mail : bestmd2000@amc.seoul.kr
} 
biopsy. Furthermore, to date, there are no validated clinical tools for assessing the characteristics and outcomes of iRPF [5-8].

Serum IgG4 concentration is one of the criteria for classifying IgG4-related diseases $[9,10]$. Considering that iRPF and IgG4-related diseases share some histopathological features including irregular fibrosis and lymphoplasmacytic infiltration, the utility of serum IgG subclasses can also be investigated in iRPF [11,12]. IgG is a major antibody in humoral immune response and it consists of four subclasses: IgG1, IgG2, IgG3 and IgG4 [13]. Among healthy adults, the concentrations of IgG subclasses in serum decreases according to their sequential order, i.e., IgG1 comprises $60 \% \sim 70 \%$ of the total IgG content; IgG2, 20\% 30\%; IgG3, 5\% 8\% and IgG4, $1 \% \sim 4 \%[14,15]$. A reverse serum IgG4/IgG3 ratio has been suggested to be one of the characteristics of IgG4related diseases that distinguishes it from primary sclerosing cholangitis [16]. However, there was no data regarding the association between serum IgG subclasses and iRPF. Here, we aimed to investigate the tools for predicting hydronephrosis-related outcomes in non-IgG4related iRPF using clinical features and serum IgG subclasses.

\section{MATERIALS AND METHODS}

\section{Study population}

The study included 18 iRPF patients with hydronephrosis from January 2012 to June 2019 in a tertiary referral hospital in South Korea. iRPF was diagnosed by the typical presentation of clinical characteristics and imaging findings on computed tomography (CT) or magnetic resonance imaging (MRI) [17,18]. Patients with IgG4-related disease fulfilling the 2019 classification criteria or secondary causes of RPF such as a history of radiation therapy, abdominal operation, malignancy or infection were excluded [9]. Biopsy was performed in cases with atypical presentation of iRPF to exclude secondary causes of RPF. Patients with hydronephrosis caused by ureter stone, malignancy or infection were also excluded from this study. This study was conducted in accordance with the Declaration of Helsinki principles. This study was approved by the Institutional Review Board of Asan Medical Center (IRB number: 2019-1344). The requirement for informed consent was waived because of the retrospective design.

\section{Baseline measurements and outcome}

We retrospectively reviewed the baseline clinical characteristics at the time of diagnosing hydronephrosis, including age, symptoms, smoking habits and comorbidities. The laboratory findings including serum creatinine, erythrocyte sedimentation rate (ESR), C-reactive protein (CRP), and complete blood count were recorded. Serum levels of IgG subclasses such as IgG1 (reference value: 365 941 mg/dL), IgG2 (reference value: 165 545 $\mathrm{mg} / \mathrm{dL}$ ), IgG3 (reference value: $32 \sim 116 \mathrm{mg} / \mathrm{dL}$ ) and IgG4 (reference value: $6 \sim 121 \mathrm{mg} / \mathrm{dL}$ ) were also measured while diagnosing hydronephrosis.

Hydronephrosis, defined as dilatation of the drainage system of the kidney such as the calyces, infundibular and pelvis, was confirmed by assessing the images obtained via CT and MRI [19]. Medications such as glucocorticoid and methotrexate were documented. Surgical intervention included ureteral stenting, percutaneous nephrostomy and an open surgical procedure.

To assess the early outcomes of hydronephrosis, we reviewed hydronephrosis status on follow-up images taken 6 months after diagnosis. We used the Society of Fetal Urology (SFU) classification to assess the degree of hydronephrosis, and defined the improvement of hydronephrosis as a complete or partial improvement of all hydronephrosis in a patient (a reduction by at least $1 \mathrm{SFU}$ grade) [20]. Patients were classified into two groups for evaluating the factors associated with the outcomes: patients with or without an improvement in hydronephrosis at 6 months.

\section{Statistical analysis}

Variables were represented as median (interquartile range [IQR]) values for continuous variables and number (\%) for categorical variables. Chi-square test or Fisher's exact test were used to assess the differences among categorical variables. Continuous variables were compared using Mann-Whitney U-test. Univariate logistic regression analysis was conducted to assess odds ratio (OR) and 95\% confidence interval (CI) for the improvement of hydronephrosis. p-values of $<0.05$ were considered to be statistically significant.

All analyses were conducted using IBM SPSS Statistics for Windows v25.0 (IBM Corp., Armonk, NY, USA). 


\section{RESULTS}

\section{Clinical and laboratory characteristics}

The clinical and laboratory characteristics of 18 iRPF patients at the time of hydronephrosis presentation are summarized in Table 1. Abdominal aorta involvement was most frequently observed, as identified in 10 patients (55.6\%). Six patients $(33.3 \%)$ exhibited ureter involvement alone. The median serum creatinine level was 1.07
(IQR $0.82 \sim 2.54) \mathrm{mg} / \mathrm{dL}$; median ESR and CRP levels were 52 (IQR $21 \sim 64) \mathrm{mm} / \mathrm{h}$ and 0.63 (IQR $0.23 \sim 5.11$ ) $\mathrm{mg} / \mathrm{dL}$; median serum IgG1, IgG2, IgG3 and IgG4 levels were 844 (IQR $734 \sim 1,001$ ) mg/dL, 559 (IQR $497 \sim 757$ ) $\mathrm{mg} / \mathrm{dL}, 66$ (IQR 42 86) mg/dL and 68 (IQR 39 134) $\mathrm{mg} / \mathrm{dL}$. Serum IgG4 level was elevated above upper normal limit (UNL) in $4(22.2 \%)$ patients and no patient had a serum IgG4 level of $>5 \times$ UNL. Tissue biopsy was performed in 6 patients using ureteroscopy. Five patients

Table 1. Baseline clinical and laboratory characteristics in iRPF patients according to the short-term outcomes of hydronephrosis

\begin{tabular}{|c|c|c|c|c|}
\hline Characteristics & $\begin{array}{l}\text { All patients } \\
\quad(\mathrm{n}=18)\end{array}$ & $\begin{array}{l}\text { With improvement } \\
\qquad(\mathrm{n}=8)\end{array}$ & $\begin{array}{l}\text { Without improvement } \\
\qquad(\mathrm{n}=10)\end{array}$ & $p$-value \\
\hline \multicolumn{5}{|l|}{ Clinical characteristics } \\
\hline Sex, male & $14(77.8)$ & $5(62.5)$ & $9(90)$ & 0.275 \\
\hline Age (yr) & $62(54 \sim 65)$ & $62(57 \sim 62)$ & $61(52 \sim 67)$ & 0.897 \\
\hline Symptoms & $16(88.9)$ & 7 (87.5) & $9(90)$ & 1.000 \\
\hline Flank pain & 7 (38.9) & $5(62.5)$ & $2(20)$ & 0.145 \\
\hline Abdominal pain & $6(33.3)$ & $1(12.5)$ & $5(50)$ & 0.152 \\
\hline Urinary frequency & $2(11.1)$ & $1(12.5)$ & $1(10)$ & 1.000 \\
\hline Leg edema & $4(22.2)$ & $1(12.5)$ & $3(30)$ & 0.588 \\
\hline Smoker & $11(61.1)$ & $5(62.5)$ & $6(60)$ & 1.000 \\
\hline Diabetes mellitus & $2(11.1)$ & $0(0)$ & $2(20)$ & 0.477 \\
\hline Hypertension & $10(55.6)$ & $4(50)$ & $6(60)$ & 1.000 \\
\hline \multicolumn{5}{|l|}{ Hydronephrosis } \\
\hline Right & $17(94.4)$ & 7 (87.5) & $10(100)$ & 0.444 \\
\hline Left & $15(83.3)$ & 7 (87.5) & $8(80)$ & 1.000 \\
\hline Bilateral & $14(77.8)$ & $6(75)$ & $8(80)$ & 1.000 \\
\hline \multicolumn{5}{|l|}{ Distribution of involvement } \\
\hline Ureter only & $6(33.3)$ & $1(12.5)$ & $5(50)$ & 0.152 \\
\hline Abdominal aorta & $10(55.6)$ & 7 (87.5) & $3(30)$ & 0.025 \\
\hline Peritoneum & $2(11.1)$ & $0(0)$ & $2(20)$ & 0.477 \\
\hline \multicolumn{5}{|l|}{ Laboratory findings } \\
\hline Serum creatinine $(\mathrm{mg} / \mathrm{dL})$ & $1.07(0.82 \sim 2.54)$ & $0.98(0.83 \sim 3.56)$ & $1.12(0.72 \sim 2.54)$ & 1.000 \\
\hline GFR $\left(\mathrm{mL} / \mathrm{min} / 1.73 \mathrm{~m}^{2}\right)$ & $76(23 \sim 86)$ & $78(18 \sim 82)$ & $72(26 \sim 95)$ & 0.573 \\
\hline WBC $\left(\times 10^{3} / \mathrm{mm}^{3}\right)$ & $7.25(5.40 \sim 8.51)$ & $6.20(5.13 \sim 7.33)$ & $7.85(6.53 \sim 8.61)$ & 0.146 \\
\hline Hemoglobin (g/dL) & $11.9(10.7 \sim 13.6)$ & $12.4(11.7 \sim 13.7)$ & $11.1(10.2 \sim 13.1)$ & 0.173 \\
\hline $\mathrm{ESR}(\mathrm{mm} / \mathrm{h})$ & $52(21 \sim 64)$ & $56(36 \sim 61)$ & $32(16 \sim 75)$ & 0.460 \\
\hline $\mathrm{CRP}(\mathrm{mg} / \mathrm{dL})$ & $0.63(0.23 \sim 5.11)$ & $0.66(0.22 \sim 3.05)$ & $0.58(0.23 \sim 10.31)$ & 0.965 \\
\hline \multicolumn{5}{|c|}{ Serum IgG subclasses levels (mg/dL) } \\
\hline $\operatorname{lgG1}$ & $844(734 \sim 1,001)$ & $902(798 \sim 1,027)$ & $806(669 \sim 951)$ & 0.408 \\
\hline $\operatorname{lgG} 2$ & $559(497 \sim 757)$ & $559(407 \sim 639)$ & $656(524 \sim 960)$ & 0.173 \\
\hline $\operatorname{lgG} 3$ & $66(42 \sim 86)$ & $61(28 \sim 91)$ & $66(45 \sim 87)$ & 0.633 \\
\hline $\operatorname{lgG} 4$ & $68(39 \sim 134)$ & $107(55 \sim 158)$ & $55(36 \sim 134)$ & 0.408 \\
\hline IgG4 > UNL, n (\%) & $4(22.2)$ & $2(25.0)$ & $2(20)$ & 1.000 \\
\hline \multicolumn{5}{|l|}{ Serum IgG subclasses ratios } \\
\hline IgG4/IgG1 & $0.08(0.06 \sim 0.13)$ & $0.10(0.06 \sim 0.20)$ & $0.07(0.05 \sim 0.11)$ & 0.315 \\
\hline $\operatorname{lgG} 4 / \operatorname{lgG} 2$ & $0.13(0.08 \sim 0.23)$ & $0.19(0.13 \sim 0.28)$ & $0.09(0.06 \sim 0.15)$ & 0.027 \\
\hline $\operatorname{lgG} 4 / \operatorname{lgG} 3$ & $1.09(0.69 \sim 2.47)$ & $2.06(1.24 \sim 2.51)$ & $0.90(0.51 \sim 1.98)$ & 0.122 \\
\hline $\operatorname{lgG} 4 / \operatorname{lgG} 3>1$ & $10(55.6)$ & 7 (87.5) & $3(30)$ & 0.025 \\
\hline
\end{tabular}

Data are expressed as number (\%) or median (interquartile range). iRPF: idiopathic retroperitoneal fibrosis, GFR: glomerular filtration rate, WBC: white blood cell, ESR: erythrocyte sedimentation rate, CRP: C-reactive protein, UNL: upper normal limit. 
showed chronic and/or acute inflammation without meeting the immunostaining criteria. One patient had dense lymphoplasmacytic infiltration with fibrosis, but no obliterative phlebitis and storiform fibrosis, and IgG4 immunostaining was rarely observed. There was no evidence of secondary RPF in histopathology.

Among 18 iRPF patients with hydronephrosis, 8 patients (44.4\%) showed an improvement in hydronephrosis 6 months after the diagnosis of hydronephrosis. Complete improvement was observed in 6 of 18 patients (33.3\%), and the grade of hydronephrosis at diagnosis was bilateral grade 3 in two, bilateral grade 2 in one, unilateral grade 2 in two and bilateral grade 1 in one patient, respectively. To identify the predictive factors affecting short-term renal outcome of iRPF complicated with hydronephrosis, we compared baseline clinical and laboratory characteristics between with and without improvement patient groups. Abdominal aorta involvement was observed more frequently in the group with improvement than in group without improvement group $(87.5 \%$ vs. $30 \%, \mathrm{p}=0.025$ ).

Median serum IgG4 level (107 [IQR 55 158] mg/dL vs. 55 [IQR 36 134] mg/dL; $\mathrm{p}=0.408$ ) did not differ significantly between the two groups. With regard to the ratios of IgG subclasses, the median serum IgG4/IgG2 ratio was higher in the group with improvement than in the group without improvement $(0.19$ [IQR $0.13 \sim 0.28]$ vs. 0.09 [IQR $0.06 \sim 0.15$ ], $\mathrm{p}=0.027$ ) and the median serum IgG4/IgG3 ratio was not different between the two groups (2.06 [IQR 1.24 2.51] vs. 0.90 [IQR 0.51 1.98], $\mathrm{p}=0.122)$. However, the group with improvement showed a higher percentage of serum IgG4/IgG3 ratio of $>1$ than the group without improvement $(87.5 \%$ vs. $30 \%, p=0.025)$.

\section{Treatment modalities for hydronephrosis}

At 6 months after diagnosis of hydronephrosis, $14 \mathrm{pa}-$ tients $(77.8 \%)$ underwent medical or surgical treatment (Table 2). The remaining four patients received no treatment. Ten patients $(56.6 \%)$ received medical treatment, all of which were treated with glucocorticoid. Methotrexate was administered to 2 patients (11.1\%). Twelve patients (66.7\%) required surgical treatment, and ureteral stenting was performing in 11 patients $(61.1 \%)$, percutaneous nephrostomy in 7 patients $(38.9 \%)$ and ileal ureter operation in 1 patient $(5.6 \%)$. Four patients $(22.2 \%)$ received surgical intervention alone without any medication.

On comparing the treatment strategies between the groups with and without improvement, the frequency of glucocorticoid administration was higher in the group with improvement than in the group without improvement $(87.5 \%$ vs. $30 \%, p=0.025)$. There was no significant difference in the percentage of cases with surgical intervention between the two groups ( $75 \%$ vs. $60 \%$, $\mathrm{p}=0.638$ ).

\section{Predictors of short-term outcomes of hydronephrosis in iRPF}

In univariate logistic regression analysis (Table 3), predictors for the improvement of hydronephrosis were serum IgG4/IgG3 ratio of $>1$, abdominal aorta involve-

Table 2. Medical and surgical treatments in iRPF patients according to the short-term outcomes of hydronephrosis

\begin{tabular}{lcccc}
\hline \multicolumn{1}{c}{ Treatment } & All patients & With improvement & Without improvement \\
& $(\mathrm{n}=18)$ & $(\mathrm{n}=8)$ & p-value \\
& $10(56.6)$ & $7(87.5)$ & $3(30)$ & 0.025 \\
Medical treatment & $10(56.6)$ & $7(87.5)$ & $3(30)$ & 0.025 \\
Glucocorticoid & $40(30 \sim 60)$ & $40(30 \sim 50)$ & $60(30 \sim 60)$ & 0.383 \\
Initial dose (prednisolone, mg/d)* & $2.42(1.81 \sim 3.69)$ & $2.10(1.51 \sim 3.30)$ & $3.67(1.91 \sim 4.27)$ & 0.383 \\
Cumulative dose (prednisolone, g)* & $4.5(2.3 \sim 6.0)$ & $4.2(2.1 \sim 5.1)$ & $6.0(4.5 \sim 6.0)$ & 0.183 \\
Duration of treatment (mo) & $2(11.1)$ & $2(25)$ & $0(0)$ & 0.183 \\
Methotrexate & $12(66.7)$ & $6(75)$ & $6(60)$ & 0.638 \\
Surgical intervention & $11(61.1)$ & $6(75)$ & $5(50)$ & 0.367 \\
Ureteral stenting & $7(38.9)$ & $3(37.5)$ & $4(40)$ & 1.000 \\
Percutaneous nephrostomy & $1(5.6)$ & $0(0)$ & $1(10)$ & 1.000 \\
Oppen surgical procedure & $4(22.2)$ & $1(12.5)$ & $3(30)$ & 0.588 \\
Surgical intervention only & & & \\
\hline
\end{tabular}

Data are expressed as number $(\%)$ or median (interquartile range). iRPF: idiopathic retroperitoneal fibrosis. ${ }^{*}$ In patients treated with glucocorticoid. 
Table 3. Univariate logistic regression analysis for the predictors of hydronephrosis improvement in iRPF patients

\begin{tabular}{lccc}
\hline \hline Variables & Odds ratio & $95 \%$ confidence interval & $p$-value \\
\hline Serum IgG subclasses ratios & & & 0.891 \\
IgG4/IgG1 & 1.91 & $0.0001 \sim 19,917.22$ & 0.109 \\
IgG4/IgG2 & $11,025.95$ & $0.13 \sim 974,570,479.54$ & 0.523 \\
IgG4/IgG3 & 1.20 & $0.69 \sim 2.10$ & 0.028 \\
IgG4/lgG3 $>1$ & 16.33 & $1.35 \sim 197.77$ & 0.028 \\
Glucocorticoid treatment & 16.33 & $1.35 \sim 197.77$ & 0.028 \\
Abdominal aorta involvement & 16.33 & $1.35 \sim 197.77$ & \\
\hline
\end{tabular}

iRPF: idiopathic retroperitoneal fibrosis.

ment and glucocorticoid treatment (OR 16.33, 95\% CI 1.35 197.77, $\mathrm{p}=0.028$, respectively). However, serum $\mathrm{IgG} 4 / \mathrm{IgG} 2$ ratio was not significantly associated with an improvement in hydronephrosis (OR 11,025.95, 95\% CI $0.13 \sim 974,570,479.54, \mathrm{p}=0.109$ ).

Four patients with a serum IgG4 level above UNL showed an obvious serum IgG4/IgG3 ratio of $>1$. Interestingly, when we analysed 14 patients with a normal serum IgG4 level, 6 patients (42.9\%) showed an improvement in hydronephrosis and the proportion of patients with a serum IgG4/IgG3 ratio of $>1$ was higher in the group with improvement than in the group without improvement ( $83.3 \%$ vs. $12.5 \%, p=0.026)$. Furthermore, the median serum IgG4/IgG3 ratio was also higher in the group with improvement than in the group without improvement (1.73 [IQR 0.97 2.20] vs. 0.80 [IQR 0.43 0.96], $\mathrm{p}=0.029)$.

\section{DISCUSSION}

iRPF is generally known to have a good prognosis. However, persistent hydronephrosis may lead to poor renal outcomes including chronic kidney disease and renal atrophy $[7,21]$. A proper intervention, such as medical and surgical treatments, is necessary for an early relief of ureteral obstruction [22]. In the present study, hydronephrosis improved in almost half of the patients on short-term follow-up, consistent with the iRPF outcome reported in previous studies $[23,24]$. Besides the clinical features associated with iRPF outcomes, short-term follow-up images revealed that a reverse serum IgG4/IgG3 ratio was also associated with an improvement in hydronephrosis. This pattern was observed even in patients with a normal serum IgG4 level. Thus, our results suggested that the serum IgG4/IgG3 ratio is a marker for predicting the short-term outcomes of iRPF patients with hydronephrosis.

We found that a quarter of the patients had a serum IgG4 level above UNL which may share the features with IgG4-related RPF. Zen et al. [25] reported that the levels of serum IgG4 and serum IgG4/IgG ratio were higher in biopsy-proven IgG4-related RPF than in non-IgG4-related RPF. Several reports suggested that a high serum IgG4 level was associated with a high disease activity and high relapse rate of IgG4-related disease [26,27]. However, we found that there was no difference in the proportion of patients with a serum IgG4 level above UNL between the groups with and without improvement. Our result was similar to a previous prospective study that failed to demonstrate a significant association between an elevated serum IgG4 level and the success of tamoxifen treatment in iRPF [28].

Little is known about the levels of serum IgG subclasses, except IgG4, in iRPF. Wallace et al. [29] found that $51 \%$ of the patients with an active IgG4-related disease had an elevated serum IgG4 level and $26 \%$ of the patients had an elevated serum IgG3 level. In our study, serum IgG3 level did not differ between the with and without improvement groups, and the median serum IgG3 levels were $54 \mathrm{mg} / \mathrm{dL}$ and $68 \mathrm{mg} / \mathrm{dL}$ in the elevated serum IgG4 and normal serum IgG4 groups, respectively. Furthermore, there were only two patients with a serum IgG3 level above UNL, which indicates that a reverse serum IgG4/IgG3 ratio may be useful for predicting renal outcomes in iRPF, especially for patients with a normal serum IgG4 level.

Medical treatment with glucocorticoid was one of the predictive factors that differed between patients with and without improvement. Glucocorticoid administration is considered to be an effective treatment strategy for achieving remission of iRPF $[30,31]$. Abdominal aorta involvement was also associated with an improvement in hydronephrosis. Previous studies have reported that 
IgG4-related RPF tends to show more extra-retroperitoneal manifestations than non-IgG4-related RPF $[4,32]$. In IgG4-related diseases, patients with a high serum IgG4 level may show multiple organ involvement [27]. Ozawa et al. [33] demonstrated that patients with IgG4-related diseases who had a complication of periaortitis had more inflammatory characteristics, including a high serum IgG4 level, than those who did not have periaortitis. Based on these previous results, glucocorticoid response and the presence of abdominal aorta involvement in iRPF patients with ureter involvement may suggest that patients are affected by the similar mechanisms of IgG4-related RPF.

This study had several limitations. First, because of the retrospective nature of the study, clinical data available may have been limited. Second, despite the rarity of this disease, a limited number of cases was not enough for performing multivariate logistic regression analysis to verify the factors associated with the disease outcomes. Finally, tissue biopsy was not performed in all patients, which makes it difficult to define exclusion of IgG4-related RPF. However, investigating the utility of a clinical predictor is valuable in itself because of the challenges in obtaining pathological information in a real clinical setting.

\section{CONCLUSION}

Reverse serum IgG4/IgG3 ratio, glucocorticoid treatment and involvement of the abdominal aorta was associated with a favourable renal outcome in iRPF patients with hydronephrosis. With regard to the predictors related to the prognosis of iRPF with hydronephrosis, the serum IgG4/IgG3 ratio might be a useful marker.

\section{ACKNOWLEDGMENTS}

This work was supported by a grant from the Asan Institute for Life Sciences, Asan Medical Center, Korea (2020IL0037).

\section{CONFLICT OF INTEREST}

No potential conflict of interest relevant to this article was reported.

\section{AUTHOR CONTRIBUTIONS}

Y.G.K. and B.S.H. were involved in conception and design of study. S.J.C. and Y.G.K. were contributed to acquisition, analysis and interpretation of data. All authors were involved in drafting and revising the manuscript critically for important intellectual content and final approval of the version to be published. J.S.O., S.H., C.K.L., and B.Y. participated in the acquisition of data.

\section{REFERENCES}

1. Vaglio A, Salvarani C, Buzio C. Retroperitoneal fibrosis. Lancet 2006;367:241-51.

2. Baker LR, Mallinson WJ, Gregory MC, Menzies EA, Cattell WR, Whitfield HN, et al. Idiopathic retroperitoneal fibrosis. A retrospective analysis of 60 cases. Br J Urol 1987;60: 497-503.

3. Vaglio A, Maritati F. Idiopathic retroperitoneal fibrosis. J Am Soc Nephrol 2016;27:1880-9.

4. Khosroshahi A, Carruthers MN, Stone JH, Shinagare S, Sainani N, Hasserjian RP, et al. Rethinking Ormond's disease: "idiopathic" retroperitoneal fibrosis in the era of IgG4-related disease. Medicine (Baltimore) 2013;92:82-91.

5. Magrey MN, Husni ME, Kushner I, Calabrese LH. Do acute-phase reactants predict response to glucocorticoid therapy in retroperitoneal fibrosis? Arthritis Rheum 2009; 61:674-9.

6. Pelkmans LG, Aarnoudse AJ, Hendriksz TR, van Bommel EF. Value of acute-phase reactants in monitoring disease activity and treatment response in idiopathic retroperitoneal fibrosis. Nephrol Dial Transplant 2012;27:2819-25.

7. Zhao J, Li J, Zhang Z. Long-term outcomes and predictors of a large cohort of idiopathic retroperitoneal fibrosis patients: a retrospective study. Scand J Rheumatol 2019;48:239-45.

8. Forestier A, Buob D, Mirault T, Puech P, Gnemmi V, Launay $D$, et al. No specific imaging pattern can help differentiate IgG4-related disease from idiopathic retroperitoneal fibrosis: 18 histologically proven cases. Clin Exp Rheumatol 2018;36:371-5.

9. Wallace ZS, Naden RP, Chari S, Choi HK, Della-Torre E, Dicaire JF, et al.; Members of the ACR/EULAR IgG4-RD Classification Criteria Working Group. The 2019 American College of Rheumatology/European League Against Rheumatism classification criteria for IgG4-related disease. Ann Rheum Dis 2020;79:77-87.

10. Xia CS, Fan CH, Liu YY. Diagnostic performances of serum IgG4 concentration and IgG4/IgG ratio in IgG4-related disease. Clin Rheumatol 2017;36:2769-74.

11. Stone JH, Zen Y, Deshpande V. IgG4-related disease. N Engl J Med 2012;366:539-51.

12. Corradi D, Maestri R, Palmisano A, Bosio S, Greco P, Manenti L, et al. Idiopathic retroperitoneal fibrosis: clinicopathologic features and differential diagnosis. Kidney Int 2007;72:742-53.

13. Vidarsson G, Dekkers G, Rispens T. IgG subclasses and allotypes: from structure to effector functions. Front Immunol 
2014;5:520.

14. Brito-Zerón P, Ramos-Casals M, Bosch X, Stone JH. The clinical spectrum of IgG4-related disease. Autoimmun Rev 2014;13:1203-10.

15. Stone JH, Brito-Zerón P, Bosch X, Ramos-Casals M. Diagnostic approach to the complexity of IgG4-related disease. Mayo Clin Proc 2015;90:927-39.

16. Boonstra K, Culver EL, de Buy Wenniger LM, van Heerde MJ, van Erpecum KJ, Poen AC, et al. Serum immunoglobulin G4 and immunoglobulin G1 for distinguishing immunoglobulin G4-associated cholangitis from primary sclerosing cholangitis. Hepatology 2014;59:1954-63.

17. Cronin CG, Lohan DG, Blake MA, Roche C, McCarthy P, Murphy JM. Retroperitoneal fibrosis: a review of clinical features and imaging findings. AJR Am J Roentgenol 2008; 191:423-31.

18. Ha YJ, Jung SJ, Lee KH, Lee SW, Lee SK, Park YB. Retroperitoneal fibrosis in 27 Korean patients: single center experience. J Korean Med Sci 2011;26:985-90.

19. Robins SA, Fischmann J. Hydronephrosis; a radiologic classification based on anatomical variations. Radiology 1948; 50:632-8.

20. Kim J, Park S, Hwang H, Kim JW, Cheon SH, Park S, et al. Comparison of surgical outcomes between dismembered pyeloplasty with or without ureteral stenting in children with ureteropelvic junction obstruction. Korean J Urol 2012;53:564-8.

21. Soliman SA, Shokeir AA, Mosbah A, Abol-Enein H, Barakat $\mathrm{N}$, Abou-Bieh E, et al. Recoverability of renal function after relief of chronic partial unilateral ureteric obstruction: the effect of an angiotensin-converting enzyme inhibitor (enalapril). Arab J Urol 2011;9:67-71.

22. Fry AC, Singh S, Gunda SS, Boustead GB, Hanbury DC, McNicholas TA, et al. Successful use of steroids and ureteric stents in 24 patients with idiopathic retroperitoneal fibrosis: a retrospective study. Nephron Clin Pract 2008;108: c213-20.

23. Kermani TA, Crowson CS, Achenbach SJ, Luthra HS. Idiopathic retroperitoneal fibrosis: a retrospective review of clinical presentation, treatment, and outcomes. Mayo Clin Proc 2011;86:297-303.
24. Yachoui R, Sehgal R, Carmichael B. Idiopathic retroperitoneal fibrosis: clinicopathologic features and outcome analysis. Clin Rheumatol 2016;35:401-7.

25. Zen Y, Onodera M, Inoue D, Kitao A, Matsui O, Nohara T, et al. Retroperitoneal fibrosis: a clinicopathologic study with respect to immunoglobulin G4. Am J Surg Pathol 2009;33:1833-9.

26. Tabata T, Kamisawa T, Takuma K, Egawa N, Setoguchi K, Tsuruta K, et al. Serial changes of elevated serum IgG4 levels in IgG4-related systemic disease. Intern Med 2011;50: 69-75.

27. Culver EL, Sadler R, Simpson D, Cargill T, Makuch M, Bateman AC, et al. Elevated serum IgG4 levels in diagnosis, treatment response, organ involvement, and relapse in a prospective IgG4-related disease UK cohort. AmJ Gastroenterol 2016;111:733-43.

28. Pelkmans LG, Hendriksz TR, Westenend PJ, Vermeer HJ, van Bommel EFH. Elevated serum IgG4 levels in diagnosis and treatment response in patients with idiopathic retroperitoneal fibrosis. Clin Rheumatol 2017;36:903-12.

29. Wallace ZS, Deshpande V, Mattoo H, Mahajan VS, Kulikova M, Pillai S, et al. IgG4-related disease: clinical and laboratory features in one hundred twenty-five patients. Arthritis Rheumatol 2015;67:2466-75.

30. van Bommel EF, Siemes C, Hak LE, van der Veer SJ, Hendriksz TR. Long-term renal and patient outcome in idiopathic retroperitoneal fibrosis treated with prednisone. Am J Kidney Dis 2007;49:615-25.

31. van der Bilt FE, Hendriksz TR, van der Meijden WA, Brilman LG, van Bommel EF. Outcome in patients with idiopathic retroperitoneal fibrosis treated with corticosteroid or tamoxifen monotherapy. Clin Kidney J 2016;9:184-91.

32. Rossi GM, Rocco R, Accorsi Buttini E, Marvisi C, Vaglio A. Idiopathic retroperitoneal fibrosis and its overlap with IgG4-related disease. Intern Emerg Med 2017;12:287-99.

33. Ozawa M, Fujinaga Y, Asano J, Nakamura A, Watanabe T, Ito $\mathrm{T}$, et al. Clinical features of IgG4-related periaortitis/ periarteritis based on the analysis of 179 patients with IgG4-related disease: a case-control study. Arthritis Res Ther 2017;19:223. 